\title{
Mineralocorticoid hypertension and hypokalaemia induced by posaconazole
}

\section{Charlotte Boughton', David Taylor², Lea Ghataore², Norman Taylor ${ }^{2}$ and Benjamin C Whitelaw'}

'Endocrinology Department and 2Department of Clinical Biochemistry (Viapath), King's College Hospital NHS Foundation Trust, London, UK
Correspondence

should be addressed

to $C$ Boughton

Email

charlotte.boughton@nhs.net

\section{Summary}

We describe severe hypokalaemia and hypertension due to a mineralocorticoid effect in a patient with myelodysplastic syndrome taking posaconazole as antifungal prophylaxis. Two distinct mechanisms due to posaconazole are identified: inhibition of $11 \beta$ hydroxylase leading to the accumulation of the mineralocorticoid hormone 11-deoxycorticosterone (DOC) and secondly, inhibition of $11 \beta$ hydroxysteroid dehydrogenase type 2 (11ßHSD2), as demonstrated by an elevated serum cortisol-to-cortisone ratio. The effects were ameliorated by spironolactone. We also suggest that posaconazole may cause cortisol insufficiency. Patients taking posaconazole should therefore be monitored for hypokalaemia, hypertension and symptoms of hypocortisolaemia, at the onset of treatment and on a monthly basis. Treatment with mineralocorticoid antagonists (spironolactone or eplerenone), supplementation of glucocorticoids (e.g. hydrocortisone) or dose reduction or cessation of posaconazole should all be considered as management strategies.

\section{Learning points:}

- Combined hypertension and hypokalaemia are suggestive of mineralocorticoid excess; further investigation is appropriate.

- If serum aldosterone is suppressed, then further investigation to assess for an alternative mineralocorticoid is appropriate, potentially using urine steroid profiling and/or serum steroid panelling.

- Posaconazole can cause both hypokalaemia and hypertension, and we propose that this is due to two mechanisms - both $11 \beta$ hydroxylase inhibition and $11 \beta$ HSD2 inhibition.

- Posaconazole treatment may lead to cortisol insufficiency, which may require treatment; however, in this clinical case, the effect was mild.

- First-line treatment of this presentation would likely be use of a mineralocorticoid antagonist.

- Patients taking posaconazole should be monitored for hypertension and hypokalaemia on initiation and monthly thereafter.

\section{Background}

This case demonstrates hypertension and hypokalaemia due to a mineralocorticoid effect induced by posaconazole. Two distinct mechanisms have been identified: inhibition of $11 \beta$ hydroxylase leading to the accumulation of the mineralocorticoid hormone 11-deoxycorticosterone (DOC) and inhibition of $11 \beta$ hydroxysteroid dehydrogenase type 2 (11ßHSD2), as demonstrated by an elevated serum cortisol-to-cortisone ratio. The effects were ameliorated by spironolactone.

Although not clinically relevant to this case, we have also demonstrated that posaconazole may cause cortisol insufficiency. 


\section{Case presentation}

A previously well 67-year-old man was diagnosed with myelodysplastic syndrome in 2014 (refractory cytopaenia with multilineage dysplasia-RCMD). He was treated with chemotherapy (daunorubicin and cytarabine), which was complicated by a fungal chest infection. Antifungal therapy, initially intravenous ambisome and caspofungin, was given. Subsequently, he was started on prophylactic posaconazole $200 \mathrm{mg}$ TDS. His serum potassium decreased at this point, but this was attributed to gastrointestinal losses from diarrhoea and was not further investigated. He was normotensive at this time with systolic blood pressure in the range of $120-130 \mathrm{mmHg}$.

During 2015, he had a second cycle of chemotherapy and a subsequent stem cell transplant, complicated by reactivation of TB (bone marrow and mediastinal glands), which was treated with quadruple anti-tuberculosis therapy. Posaconazole was briefly interrupted twice during this year: for intravenous antifungal treatment (ambisome/caspofungin) and during the stem cell transplant. Prophylactic posaconazole was stopped completely at the end of 2015 , as he was in remission. Serum potassium normalised.

Over the following 12 months (2016), while not taking posaconazole, potassium remained within the normal range and the patient continued to be normotensive.

In January 2017, there was a relapse of myelodysplastic syndrome, treated with chemotherapy (Azacitidine), and posaconazole $300 \mathrm{mg}$ OD was recommenced. Posaconazole was interrupted for an admission with neutropenic sepsis and decompensated heart failure but then restarted on discharge in February 2017. At this point, he was also taking ramipril and bisoprolol, commenced for heart failure.

Hypokalaemia $(2.7-2.9 \mathrm{mmol} / \mathrm{L})$ was noted shortly after this and his doses of antihypertensive medication were increased to control elevated blood pressure (systolic blood pressure $150-170 \mathrm{mmHg}$ ). There had been no previous history of hypertension. Hypokalaemia was managed with oral and intermittent intravenous potassium replacement, but despite these measures, he required admission for symptomatic hypokalaemia $(2.4 \mathrm{mmol} / \mathrm{L})$. No other sources of potassium loss were identified on clinical history; he was not on any medications causing potassium loss at this time, had no diarrhoea or vomiting and had a normal dietary intake of potassium.

He was at this point referred to the endocrine team. On clinical assessment, he was hypertensive but had no symptoms or clinical features of Cushing's or other endocrinopathy. Investigations were undertaken to assess for suspected mineralocorticoid hypertension and hypokalaemia.

\section{Investigation}

\section{Investigations and results}

Laboratory results confirmed persistent hypokalaemia (2.7-2.9 mmol/L) with an alkalosis ( $\mathrm{pH} 7.52$ bicarbonate $32 \mathrm{mmol} / \mathrm{L}$ ). Other serum electrolytes were normal apart from mild hypomagnesaemia. Renal function was normal. Urinary potassium was very high $(59 \mathrm{mmol} / \mathrm{L})$.

Plasma renin levels (Diasorin Liaison XLimmunoassay) were undetectable $(<0.5 \mathrm{U} / \mathrm{L})$ and serum aldosterone was also very low $(32 \mathrm{pmol} / \mathrm{L})$ (normal $>140 \mathrm{pmol} / \mathrm{L}$ ). Cortisol (Siemens Centaur XPi immunoassay) at 09:00 h was $269 \mathrm{nmol} / \mathrm{L}$ and a short synacthen test $(250 \mu \mathrm{g})$ demonstrated inadequate cortisol response (baseline: $384 \mathrm{nmol} / \mathrm{L}, 30 \mathrm{~min}: 430 \mathrm{nmol} / \mathrm{L}, 60 \mathrm{~min}: 446 \mathrm{nmol} / \mathrm{L}$ ). ACTH was $118 \mathrm{ng} / \mathrm{L}$ (normal: 10-50). An overnight $1 \mathrm{mg}$ dexamethasone test showed full cortisol suppression $(<30 \mathrm{nmol} / \mathrm{L})$.

A serum steroid profile by LC-MS/MS showed (nmol/L, (normal range)) markedly elevated 11-deoxycorticosterone (DOC): 12.5 (<1.4) and 11-deoxycortisol: 63.1 (<2.7). The serum cortisol (227)-to-cortisone (14.8) ratio was raised at $15.3(1.0-10.5)$. Androstenedione was slightly elevated at $9.1 \mathrm{nmol} / \mathrm{L}(<7.0)$.

A urinary steroid profile also demonstrated marked relative increases of metabolites of DOC and 11-deoxycortisol, with lesser increases of metabolites of 17-hydroxyprogesterone. 11-Hydroxylated androgen metabolites were at very low levels relative to cortisol

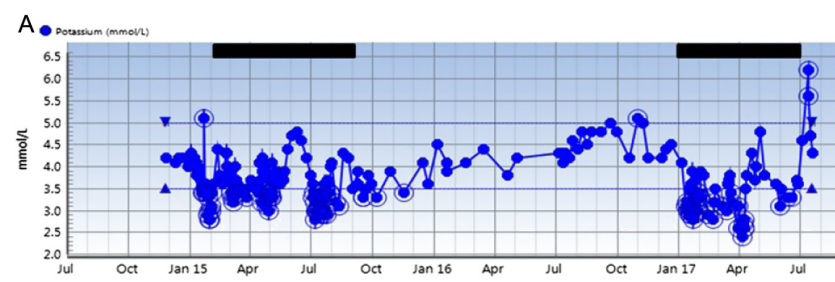

B

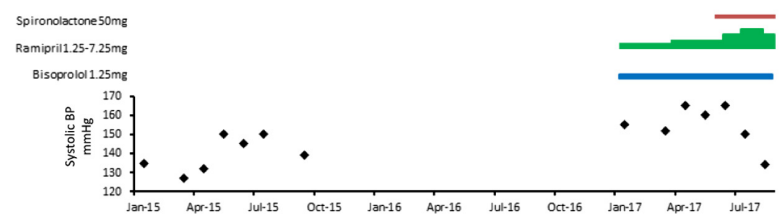

Figure 1

(A) Intermittent hypokalaemia that occurs at the times when the patient is taking posaconazole (represented by black bars). (B) Systolic blood pressure and antihypertensive medications including treatment with spironolactone. 


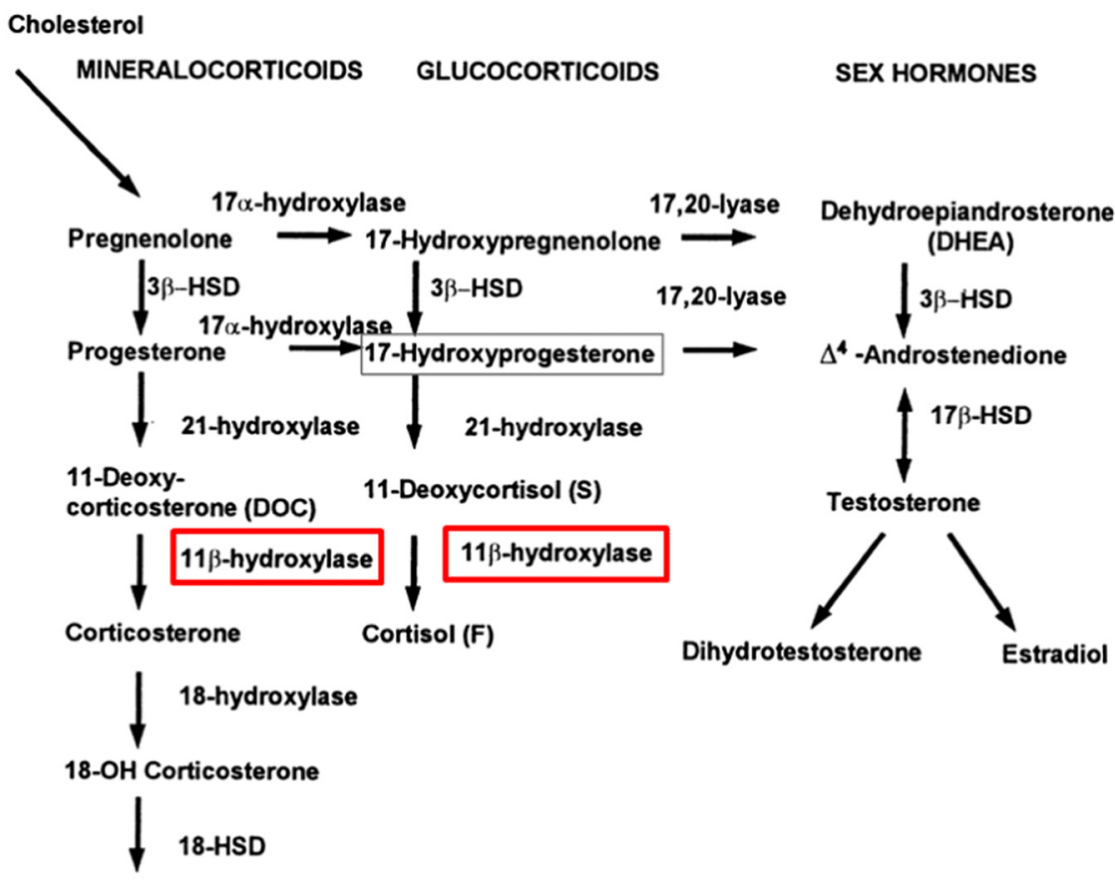

Aldosterone

metabolites. The ratio of cortisol/cortisone metabolites was normal. These findings mimic genetic $11 \beta$-hydroxylase deficiency and also use of the specific 11 1 -hydroxylase inhibitor, metyrapone.

Axial imaging to evaluate progression of his haematological malignancy noted bilateral hyperplastic adrenal glands. There were no radiological features of adrenal TB.

\section{Treatment}

The patient was commenced on spironolactone $50 \mathrm{mg}$ once daily to antagonise the mineralocorticoid effects caused by posaconazole. The hypokalaemia resolved and oral potassium supplementation was stopped (Fig. 1). His hypertension also resolved (blood pressure: $111 / 64 \mathrm{mmHg}$ ).

The patient was also advised regarding the potential for adrenal insufficiency. He was not thought to require regular hydrocortisone but advised to seek urgent medical attention in the event of symptoms suggestive of hypocortisolaemia.

The patient subsequently stopped posaconazole in agreement with the haematologists. His myelodysplasia is being managed with donor lymphocyte infusions (DLI). He is now transfusion dependent with hyperferritinaemia requiring iron chelation therapy.

\section{Figure 2}

Steroid synthesis pathway. Inhibition of $11 \beta$ hydroxylase (highlighted) results in increase of DOC and 11-deoxycortisol (S), due to attenuation of cortisol feedback inhibition of the hypothalamo-pituitary-adrenal axis.

\section{Outcome and follow-up}

The overall diagnosis is posaconazole-induced mineralocorticoid hypertension and hypokalaemia. The investigations demonstrate that there are two mechanisms by which posaconazole cause this:

The first mechanism is $11 \beta$ hydroxylase inhibition leading to accumulation of the precursor hormone, DOC (Fig. 2). This is demonstrated by both the serum and urine steroid assessments. This hormone has a mineralocorticoid effect and can account for the hypertension and hypokalaemia observed.

The second mechanism is inhibition of $11 \beta$ hydroxysteroid dehydrogenase type 2 (11ßHSD2), the enzyme converting cortisol to cortisone in the distal convoluted renal tubule (Fig. 3). This enzyme functions at this site to deactivate cortisol and prevent it from binding and activating the type 1 mineralocorticoid receptor.

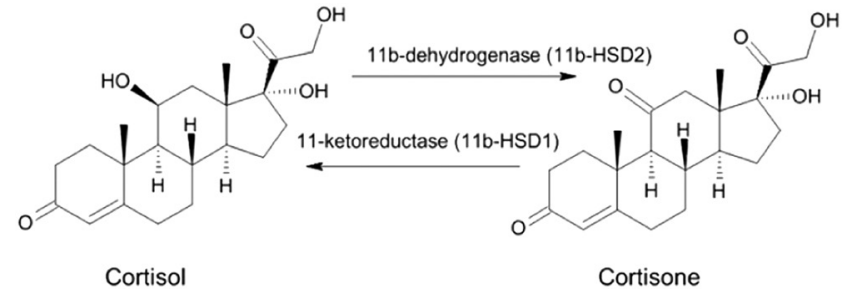

Figure 3

Cortisol to cortisone conversion by $11 \beta \mathrm{HSD}$ type 2 . 


\section{Discussion}

This case demonstrates hypertension and hypokalaemia due to a mineralocorticoid effect induced by posaconazole. Previous clinical trial data have demonstrated hypokalaemia occurring in $22 \%$ and hypertension in $11 \%$ of patients receiving the $300 \mathrm{mg}$ daily oral posaconazole (1). However, in these studies, hypokalaemia was attributed to the vomiting, which was reported in 13\% and/or diarrhoea, which was reported in $29 \%$ of the haematological malignancy population.

Posaconazole-induced

hypokalaemia

and hypertension due to presumed disruption of the steroid biosynthesis pathway has been previously described $(2,3$, 4). Thompson and coworkers went on to further investigate the mechanism and suggest that the mineralocorticoid effect is due solely to $11 \beta \mathrm{HSD} 2$ inhibition (4).

Cortisol is converted to inactive cortisone by $11 \beta \mathrm{HSD} 2$ (Fig. 3). Cortisone, unlike cortisol, does not bind to the mineralocorticoid receptor. The activity of $11 \beta \mathrm{HSD} 2$ is therefore essential in preventing cortisol (which circulates at 1000 fold higher concentrations than aldosterone) from binding and activating the mineralocorticoid receptor.

When the activity of $11 \beta \mathrm{HSD} 2$ is impaired, as can be seen with genetic loss-of-function mutations in the syndrome of apparent mineralocorticoid excess, even normal physiological levels of cortisol can increase the mineralocorticoid activity (5). Excessive cortisoldependent mineralocorticoid receptor activation causes hypokalaemia, hypernatraemia and water retention, leading to severe hypertension with low renin, low aldosterone and increased plasma and urinary cortisol-tocortisone ratios.

This hypothesised mechanism for the effect of posaconazole was first signalled by recent studies (6). These set out to identify potential 11ßHSD inhibitors among approved drugs: several azole antifungals were identified as active. Posaconazole (and structurally similar itraconazole) demonstrated relative selectivity for

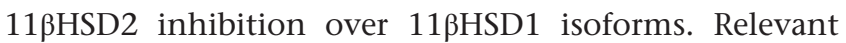
to the present study is that they were found to be much more potent in vitro than in vivo.

Regarding the biochemical evidence in our patient, an obvious increase in the ratio of urinary cortisol/cortisone metabolites was not found, but consumption of liquorice, which contains the 11ßHSD2 inhibitor, glycyrrhizic acid, in amounts that cause mineralocorticoid effects, results in only a slight change in this ratio; the ratio of cortisol/ cortisone in blood appears to be the better marker, so these findings do not negate inhibition of 11ßHSD2 in this patient, but its relative importance is difficult to judge. A useful review of some of these issues is by Ferrari and coworkers (7).

Whilst inhibition of $11 \beta \mathrm{HSD} 2$ can account for the finding of increase of cortisol relative to cortisone in serum, it does not explain the increased levels of 11-deoxycorticosterone (DOC) and 11-deoxycortisol. In the report by Thompson and coworkers, their proposed mechanism of mineralocorticoid hypertension cannot explain their own findings of increased 11-deoxycortisol, 17OHP, androstenedione and oestradiol (4). They propose that these increases of steroid precursors can be attributed to $11 \beta \mathrm{HSD} 2$ inhibition, but this is not a recognised or plausible mechanism and does not occur in genetic deficiency of $11 \beta \mathrm{HSD} 2$ (8).

Thompson and coworkers did not find an elevated 11-deoxycorticosterone (DOC) level. Unfortunately, these authors do not describe their steroid analyses. If immunometric methods were used, there is a risk that interference by structurally related steroids would obscure significance changes, especially when levels are low relative to those of the interferents. This apart, their results do support the mechanism of $11 \beta$ hydroxylase inhibition.

The genetic form of $11 \beta$ hydroxylase deficiency is characterised by glucocorticoid-responsive arterial hypertension, which is interpreted as being due to DOC increase. Taking the generally quoted mineralocorticoid potency of DOC as $1 / 40$ that of aldosterone, the value we recorded for DOC of $12.5 \mathrm{nmol} / \mathrm{L}$ equates to $313 \mathrm{pmol} / \mathrm{L}$ aldosterone, which is within the normal range for this steroid. However, as described by Vinson and coworkers (9), estimates of the potency of DOC vary, depending on the criteria used, and there is some evidence that 11-deoxycortisol also has mineralocorticoid activity, so that the relative contributions of these and cortisol to total mineralocorticoid receptor binding in this patient cannot be estimated.

The biochemical findings in both our case and the Thompson and coworkers' case can be explained by $11 \beta$ hydroxylase inhibition and the clinical and biochemical profile mimics that found in congenital adrenal hyperplasia due to $11 \beta$ hydroxylase deficiency. Therefore, we disagree with Thompson and coworkers and propose that the clinical findings in both cases can only be explained by posaconazole having a dual mechanism: inhibiting both $11 \beta \mathrm{HSD} 2$ and $11 \beta$ hydroxylase.

Structurally similar itraconazole has also been associated with a syndrome of hypertension, hypokalaemia and oedema $(10,11,12)$. The exact mechanism for this effect has not been fully 
elucidated; Antonarakis and coworkers report a dosedependent suppression of aldosterone levels with elevated corticosterone and DOC levels (12). Denolle and coworkers demonstrated low plasma renin and aldosterone levels but with normal DOC and cortisol levels (11). Ketoconazole also inhibits $11 \beta$ hydroxylase in both in vitro studies and in clinical case series in association with increased DOC and 11-deoxycortisol; cortisol levels are unchanged and corticosterone levels are inconsistently mildly elevated $(13,14)$.

\section{Conclusion}

This case report demonstrates that posaconazole caused induction of severe hypokalaemia and hypertension. Two mechanisms are identified: inhibition of both 11ßHSD2 and $11 \beta$ hydroxylase. Although not clinically relevant to this case, we have also demonstrated that posaconazole may cause cortisol insufficiency.

Patients taking posaconazole should be monitored for hypokalaemia, hypertension and symptoms of hypocortisolaemia, at the onset of treatment and on a monthly basis. Treatment with mineralocorticoid antagonists (spironolactone or eplerenone), supplementation of glucocorticoids (with hydrocortisone treatment) or dose reduction or cessation of posaconazole should all be considered as management strategies.

\section{Declaration of interest}

The authors declare that there is no conflict of interest that could be perceived as prejudicing the impartiality of the research reported.

\section{Funding}

This research did not receive any specific grant from any funding agency in the public, commercial or not-for-profit sector.

\section{Patient consent}

Written informed consent has been obtained from the patient for publication of this article and accompanying images.

\section{Author contribution statement}

$C B$ attended on the patient and wrote the first draft of the case report; D T, L G and N T analysed and interpreted the patient data; B W supervised the care of the patient and was responsible for manuscript construction and advice. All authors contributed to the final manuscript.

\section{References}

1 Posaconazole prescribing information. Merck \& Co., Inc., 2014 (available at https://www.accessdata.fda.gov/drugsatfda_docs/label/2 015/022003s018s020,0205053s002s004,0205596s001s003lbl.pdf)

2 Mahmood M, Abu Saleh O \& Sohail MR. Hypokalemia and hypertension associated with supratherapeutic posaconazole levels. Antimicrobial Agents and Chemotherapy 201761 e00019. (https://doi. org/10.1128/AAC.00019-17)

3 Martino J, Fisher BT, Bosse KR \& Bagatell R. Suspected posaconazole toxicity in a pediatric oncology patient. Pediatric Blood and Cancer 201562 1682. (https://doi.org/10.1002/pbc.25568)

4 Thompson GR, Chang D, Wittenberg RR, McHardy I \& Semrad A. In vivo $11 \beta$-hydroxysteroid dehydrogenase inhibition in PosaconazoleInduced Hypertension and Hypokalemia . Antimicrobial Agents and Chemotherapy 201761 e00760. (https://doi.org/10.1128/AAC.00760-17)

5 White PC, Mune T \& Agarwal AK. 11beta-Hydroxysteroid dehydrogenase and the syndrome of apparent mineralocorticoid excess. Endocrine Reviews 199718 135-156. (https://doi.org/10.1210/ edrv.18.1.0288)

6 Beck KR, Bachler M, Vuorinen A, Wagner S, Akram M, Griesser U, Temml V, Klusonova P, Yamaguchi H, Schuster D, et al. Inhibition of 11beta-hydroxysteroid dehydrogenase 2 by the fungicides itraconazole and posaconazole. Biochemical Pharmacology 2017130 93-103. (https://doi.org/10.1016/j.bcp.2017.01.010)

7 Ferrari P. The role of 11B-hydroxysteroid dehydrogenase type 2 in human hypertension. Biochimica et Biophysica Acta 20101802 1178-1187. (https://doi.org/10.1016/j.bbadis.2009.10.017)

8 New MI, Levine LS, Biglieri EG, Pareira J \& Ulick S. Evidence for an unidentified steroid in a child with apparent mineralocorticoid hypertension. Journal of Clinical Endocrinology and Metabolism 1977 44 924-933. (https://doi.org/10.1210/jcem-44-5-924)

9 Vinson GP. The mislabelling of deoxycorticosterone: making sense of corticosteroid structure and function. Journal of Endocrinology 2011 211 3-16. (https://doi.org/10.1530/JOE-11-0178)

10 Tomioka H, Kaneda T, Kida Y, Kaneko M, Fujii H, Hayashi M, Tomii K, Tada K, Suzuki Y \& Karino T. An open, noncomparative multicenter study of the efficacy and safety of itraconazole injections and high dose capsules in chronic pulmonary aspergillosis. Kansenshogaku Zasshi 201185 644-651. (https://doi.org/10.11150/ kansenshogakuzasshi.85.644)

11 Denolle T, Azizi M, Massart C \& Zennaro MC. Itraconazole: a new drug-related cause of hypertension. Annales De Cardiologie Et D'Angeiologie 201463 213-215. (https://doi.org/10.1016/j. ancard.2014.05.007)

12 Antonarakis ES, Heath EI, Smith DC, Rathkopf D, Blackford AL, Danila DC, King S, Frost A, Ajiboye AS, Zhao M, et al. Repurposing itraconazole as a treatment for advanced prostate cancer: a noncomparative randomized phase II trial in men with metastatic castration-resistant prostate cancer. Oncologist 201318 163-173. (https://doi.org/10.1634/theoncologist.2012-314)

13 De Coster R, Caers I, Coene MC, Amery W, Beerens D \& Haelterman C. Effects of high dose ketoconazole therapy on the main plasma testicular and adrenal steroids in previously untreated prostatic cancer patients. Clinical Endocrinology 198624 657-664. (https://doi.org/10.1111/j.1365-2265.1986.tb01662.x)

14 Engelhardt D, Dörr G, Jaspers C \& Knorr D. Ketoconazole blocks cortisol secretion in man by inhibition of adrenal 11betahydroxylase. Klinische Wochenschrift 198563 607-612. (https://doi. org/10.1007/BF01733014)

Received in final form 14 December 2017

Accepted 11 January 2018 